\title{
NAVIGATION AND REMOTE SENSING PAYLOADS AND METHODS OF THE SARVANT UNMANNED AERIAL SYSTEM
}

\author{
P. Molina ${ }^{\mathrm{a}}$, P. Fortuny ${ }^{\mathrm{a}}$, I. Colomina ${ }^{\mathrm{a}}$, M. Remy ${ }^{\mathrm{b}}$, K.A.C. Macedo ${ }^{\mathrm{b}}$, Y.R.C. Zúnigo ${ }^{\mathrm{b}}$, E. Vaz $^{\mathrm{b}}$, D. Luebeck ${ }^{\mathrm{b}}$, J. Moreira $^{\mathrm{b}}$, M. \\ Blázquez ${ }^{\mathrm{c}}$
}

${ }^{a}$ Institute of Geomatics, Carl Friedrich Gauss 11, Castelldefels, Spain

\{pere.molina, paula.fortuny, ismael.colomina\}@ideg.es

${ }^{\mathrm{b}}$ OrbiSat Remote Sensing, Campinas \& Sao José dos Campos, Brazil

\{marco-remy, karlus.macedo, yusef, eurico, dieter.luebeck\}@orbisat.com.br, joao.moreira.orbisat@gmail.com

${ }^{c}$ GeoNumerics, Josep Pla 82, Barcelona, Spain

marta.blazquez@geonumerics.com

\section{Commission I, ICWG I/5}

KEY WORDS: SAR interferometry, UAV, orientation, INS/GNSS, topographic mapping

\begin{abstract}
:
In a large number of scenarios and missions, the technical, operational and economical advantages of UAS-based photogrammetry and remote sensing over traditional airborne and satellite platforms are apparent. Airborne Synthetic Aperture Radar (SAR) or combined optical/SAR operation in remote areas might be a case of a typical "dull, dirty, dangerous" mission suitable for unmanned operation -in harsh environments such as for example rain forest areas in Brazil, topographic mapping of small to medium sparsely inhabited remote areas with UAS-based photogrammetry and remote sensing seems to be a reasonable paradigm.
\end{abstract}

An example of such a system is the SARVANT platform, a fixed-wing aerial vehicle with a six-meter wingspan and a maximumtake-of-weight of 140 kilograms, able to carry a fifty-kilogram payload. SARVANT includes a multi-band (X and P) interferometric SAR payload, as the P-band enables the topographic mapping of densely tree-covered areas, providing terrain profile information. Moreover, the combination of X- and P-band measurements can be used to extract biomass estimations. Finally, long-term plan entails to incorporate surveying capabilities also at optical bands and deliver real-time imagery to a control station.

This paper focuses on the remote-sensing concept in SARVANT, composed by the aforementioned SAR sensor and envisioning a double optical camera configuration to cover the visible and the near-infrared spectrum. The flexibility on the optical payload election, ranging from professional, medium-format cameras to mass-market, small-format cameras, is discussed as a driver in the SARVANT development. The paper also focuses on the navigation and orientation payloads, including the sensors (IMU and GNSS), the measurement acquisition system and the proposed navigation and orientation methods. The latter includes the Fast AT procedure, which performs close to traditional Integrated Sensor Orientation (ISO) and better than Direct Sensor Orientation (DiSO), and features the advantage of not requiring the massive image processing load for the generation of tie points, although it does require some Ground Control Points (GCPs). This technique is further supported by the availability of a high quality INS/GNSS trajectory, motivated by single-pass and repeat-pass SAR interferometry requirements.

\section{INTRODUCTION}

Photogrammetry with Unmanned Aerial Systems (UAS) was pioneered by W. Wester-Ebbinghaus in 1980 (WesterEbbinghaus, 1980). In recent times, the airborne photogrammetry and remote sensing community has paid considerable attention to the use of UAS, and up to present days, various types of aerial platforms and on-board remote sensors have been investigated and assessed to ultimately determine if UAS are feasible tools for remote sensing and, in case of positive answer, which platforms and on-board sensor sets optimally fits each mission needs.

As a matter of fact, several years of UAS adoption into various service, technology and research fields -we may particularly focus on photogrammetry and remote sensing, though- have outlined a clear trend based on small aerial platforms, mostly small fixed wing and multi-rotor -thus, with small payloads,- carrying small- and/or medium-format cameras and, in general, lightweight sensing payloads. The payload complexity and sophistication range from off-the-shelve amateur photographic cameras (Cho et al., 2013) to hyperspectral ones (Rufino and Moccia, 2005; Zarco-Tejada and Berni, 2012).

Thirty years after Prof. Wester-Ebbinghaus' visionary idea, thanks to the progress in electronic component miniaturization, satellite navigation, inertial navigation and digital light sensing, UAS-based photogrammetry and remote sensing is here to stay. Technologists in general, geomaticians, map makers and users, are putting considerable pressure into civil aviation authorities to let the UAS operate under well defined, safe and operationally feasible conditions. G. Petrie (Petrie, 2013) has shown how a properly regulated UAS field will create a new market, as it has happened in the United Kingdom. 
According (Cho et al., 2013), "slow set-up and adoption of regulations that allow for easy operation and commercial flights is the principal growth limiter for the industry. This is especially the case in the US (the biggest market for most applications) where no flights are allowed and even approval for flight testing in the NAS (national airspace) is very difficult to obtain." However, not everything are difficulties; the Australian Civil Aviation Safety Authority (CASA) already maintains a list of UAV operator certificate holders for activities like aerial photography, aerial surveying, aerial advertising and a number of other business areas. Already in March 2012, the Singaporean authorities gave permission for a photogrammetric UAS mapping flight over an important area of the city. And in October 2012, the French Civil Aviation Authority, the DGAC, awarded the company Delair-Tech the first authorisation ever issued to a UAV company in France, for a civil UAV to fly over $100 \mathrm{~km}$ in French airspace.

Today, UAS-based photogrammetry and remote sensing is both technically (Eisenbeiss, 2009; Remondino et al., 2011) and commercially (Petrie, 2013) feasible. In addition, other UASbased remote sensing concepts -beyond visible spectrum vision, microwave, laser ranging, etc.- are nowadays pushing up towards common practice (Zarco-Tejada and Berni, 2012; Remy et al., 2012).

The mixture of UAS-based remote-sensing concepts may be suitable -even, mandatory- specially in challenging applications as mapping of remote areas, such as the Brazilian rain forest. More in detail, the challenges faced are terrain modelling of densely forested areas; cartographic mapping of sparsely inhabited and probably unmapped areas; and environmental monitoring tasks such as biomass estimation or infra-red sensing. Needless to say, regulation compliancy and administration approval is always a challenge for UAS, although Brazil is already formalising flying permits for private and civil UAS (MundoGEO, 2013).

This paper is devoted to describe the SARVANT system, an initiative to tackle the aforementioned applications with unmanned technology, specially focusing on its varied combination of payload sensors.

\section{THE SARVANT UNMANNED AERIAL SYSTEM}

The short-term objective of the SARVANT system is to deliver a cost-effective SAR surveying solution for areas up to five hundred squared kilometers. In addition, the long-term challenge is to perform commercial mapping with both microwave and optical bands and deliver real-time aerial imagery to the ground control station through a long-range and robust data-link.

Next subsections describe the main subsystems of the SARVANT, mainly focusing on the vehicle, and the remote sensing and navigation payloads.

\subsection{Aerial vehicle}

The aerial platform of SARVANT has been designed to meet the requirements inferred by the operation of a X- and P-band interferometric SAR system flying around one kilometre above ground. The project and development of such aerial platform is currently being conducted in cooperation with two other Brazilian companies, Aeroalcool and AGX, and sponsored by the "Financiadora de Estudos e Projetos" agency (FINEP), Brazil.

Table 1 shows the main characteristics of the SARVANT aerial platform, which is depicted in Figure 1. Further details on the development of the auto-pilot and platform can be found in (Braga et al., 2012).

Table 1: UAV main characteristics

\begin{tabular}{|l|l|}
\hline Mean Flight Height & $1000 \mathrm{~m}$ \\
\hline Flight Velocity & $200 \mathrm{~km} / \mathrm{h}$ \\
\hline Stall Velocity & $72 \mathrm{~km} / \mathrm{h}$ \\
\hline Climbing rate & $2000 \mathrm{feet} / \mathrm{min}$ \\
\hline Take-off and landing distance & $250 \mathrm{~m}$ \\
\hline Maximum take-off weight & $120 \mathrm{~kg}$ \\
\hline Payload weight & $45 \mathrm{~kg}$ \\
\hline Empty weight & $35 \mathrm{~kg}$ \\
\hline Engines & $2 \times 21.5 \mathrm{HP}$ \\
\hline Wing loading & $47 \mathrm{~kg} / \mathrm{m}^{2}$ \\
\hline Autonomy & $\sim 5 \mathrm{~h}$ \\
\hline Length & $3.845 \mathrm{~m}$ \\
\hline Wing span & $6.200 \mathrm{~m}$ \\
\hline
\end{tabular}

Figure 1: The SARVANT aerial platform

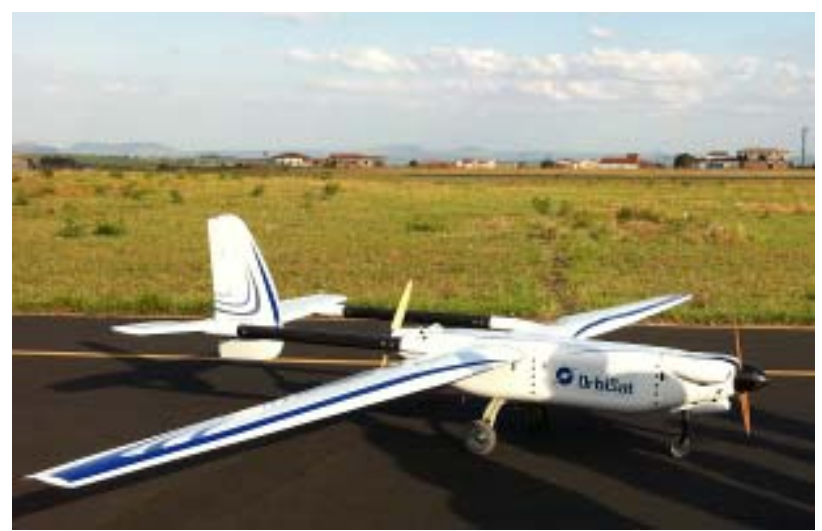

\subsection{Remote sensing payload}

$\mathrm{X}$ - and P-band synthetic aperture radar. The SAR payload consists of two P-band left- and right-looking antennas and four $\mathrm{X}$-band left- and right-looking antennas. Due to size constraints, the P-band antennas are accommodated in each horizontal stabilizer of the UAV. Yet, the X-band antennas, the radar electronics as well as the Inertial Measurement Unit (IMU) and GNSS receiver are mounted in the single physical ensemble (depicted in figure 2) and accommodated in the fuselage. Each pair of X-band antenna forms a single-pass interferometric baseline of $0.25 \mathrm{~m}$.

The on-board navigation system, based on an INS/GNSS coupling as described further on, together with the up-to-date SAR ground segment of OrbiSAR enables precise single- and repeat-pass interferometric SAR surveying. Table 2 shows the main characteristics of the X-and P-band SAR system. Again, 
further details on the construction and validation of the SAR payload of SARVANT can be found in (Remy et al., 2012).

Table 2: SAR main characteristics

\begin{tabular}{|l|l|}
\hline Volume & $\sim 0.76 \times 0.34 \times 0.36 \mathrm{~m}^{3}$ \\
\hline Weight & $30 \mathrm{~kg}$ \\
\hline Bands & P and X \\
\hline Polarization & $\mathrm{HH}$ \\
\hline Modulation & Pulsed LFM (chirp) \\
\hline Power & $\begin{array}{l}30 \mathrm{~W} \text { (peak), } 0.6 \mathrm{~W} \text { (avg.) (X-band) } \\
50 \mathrm{~W} \text { (peak), } 1.0 \mathrm{~W} \text { (avg.) (P-band) }\end{array}$ \\
\hline Range Resolution & $\begin{array}{l}\geq 0.5 \mathrm{~m} \text { (X-band) } \\
\geq 1.5 \mathrm{~m} \text { (P-band) }\end{array}$ \\
\hline Azimuth Resolution & $\begin{array}{l}\geq 0.11 \mathrm{~m} \text { (X-band) } \\
\geq 0.3 \mathrm{~m} \text { (P-band) }\end{array}$ \\
\hline Look angle & $\begin{array}{l}20 \text { to } 60 \text { deg (X-band) } \\
10 \text { to } 60 \text { deg (P-band) }\end{array}$ \\
\hline
\end{tabular}

Figure 2: $\mathrm{X}$ - and P-band synthetic aperture radar

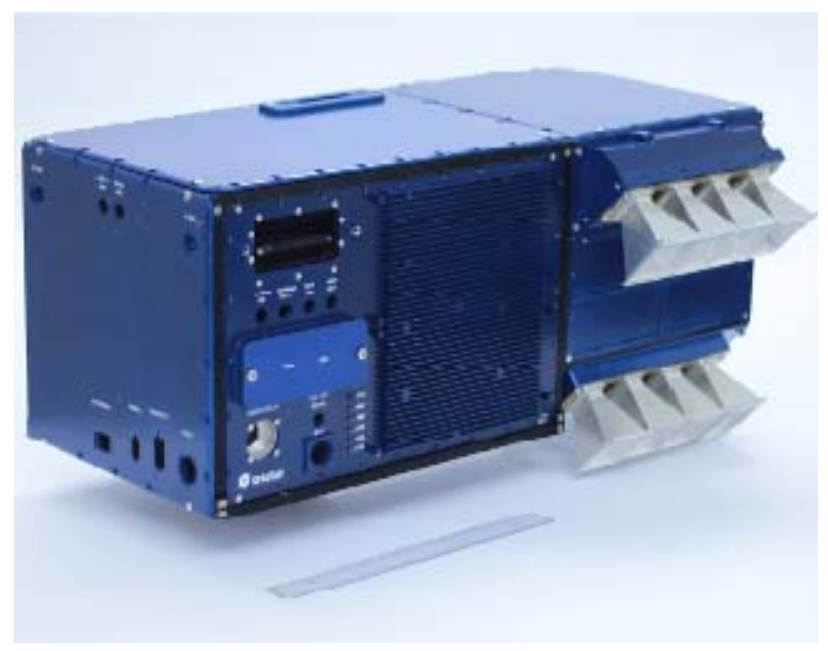

Double optical camera configuration. The traditional platform-plus-sensor concept for aerial mapping consisted in large-format cameras carried in manned airplanes, and also featured medium- and professional small-format cameras to cover the variety of requirements of each photogrammetric mission. Now, the introduction of new platforms i.e. UAVs for aerial observation has led to the miniaturization of imaging technology. Some imaging sensors have been designed as a result of specific platform developments, yet others have not been introduced specifically for the photogrammetric market but, regarding size, weight and mainly cost, they comfortably fit the requirements. In relation to the application field of the SARVANT concept, two major applications are targeted, namely cartographic and agricultural and/or environmental applications.

With respect to the cartographic application, large-format cameras are clearly out of scope of UAV-based photogrammetry. However, medium-format cameras have been sharing the photogrammetric market with large-format cameras, therefore achieving a high degree of maturation, and some are likely to meet the size and weight requirements for UAVs.
Some examples are the Trimble Aerial Camera, the Hasselblad H4D-40 and the Phase One iXA aerial camera. The latter example presents the advantage of ensuring time synchronization in multi-camera configurations by using fast synch lenses. Small-format cameras, especially amateur cameras, are somehow meant for UAV payload developers aiming at a deeper integration with on-board sensors. Besides the economic benefits, these sensors generally enable camera external control and communication at a reasonable development effort -however, one has to live with poorer image quality and more unstable calibration, which is directly translated into additional processing work, and a shorter lifetime. Regarding SARVANT, this category is considered as an interesting niche to achieve operability in an early stage. More precisely, the Mirrorless Interchangeable-Lens Cameras (MILC) are of interest due to reduced dimensions in comparison with Digital Single-Lens Reflex (DSLR) cameras and the flexibility of having a set of available lenses that compact cameras do not have. Some examples are the SONY NEX series, the Panasonic Lumix DMC and the Canon Ixus, which are of actual use for UAV systems (Manyoky et al., 2011; Strecha et al., 2012; Vallet et al., 2011).

For agricultural and/or environmental applications, both the visible and the infrared regions of the electromagnetic spectrum are commonly collected. Specificities of the targeted application mainly determine the region of the spectrum required, and therefore the use of multi-spectral or hyperspectral sensors. The former sensing technology captures information in specific portions of the spectrum and at a given resolution, which are commonly sufficient for computing the main vegetation indexes of interest. However, the latter technology is able to sense narrower spectral bands over a continuous spectral range, producing this way the spectra of all pixels in the scene. The use of these sensors provides a deeper knowledge and extraction of information. Precisely, the shortwave infrared (SWIR) has been identified as suitable to discriminate different cover types as it is associated to vegetation variations in the moisture content. Some developments are already in place suitable for small UAS, such as the multi-spectral Mini MCA family or the hyperspectral camera from Rikola Ltd., and have already been integrated in current systems (Bendig et al., 2012; C-Astral Ltd., 2013).

Therefore, the SARVANT system envisions an integration of a dual SONY NEX7 configuration. In order to cover with the agricultural applications, one of the two cameras will be modified by replacing the low-pass filter by an infrared filter allowing acquisition on the NIR range of the spectrum. In the long term, the goal is to upgrade the optical payload to mediumformat cameras for both cartographic and agricultural applications: the system would then benefit from its aforementioned enhanced performance by assuming the cost difference.

It is noteworthy that the combination of the RGB/NIR image general capabilities and, particularly, their derived vegetation indices -the Normalized Difference Vegetation Index (NDVI) being the most used of them- together with the X- and P-band SAR extends the range of applications to those of a comprehensive multispectral/geometric remote sensing system.

\subsection{Navigation payload}

In order to generate accurate heights of the terrain, both the absolute (of the aircraft trajectory) and relative positions (of the 
interferometric baseline) are required. This stringent requirements lead to the use of navigation-grade inertial measurement units (IMU) and of geodetic dual-frequency kinematic GPS receivers. The P-Band data are particularly sensitive to antenna orientation errors larger than a few centimetres.

\section{Inertial Navigation System and Global Navigation Satellite} systems. To accomplish the task of orienting the UAV i.e. estimating its position, velocity and attitude (PVA), an Inertial Navigation System (INS) is strap-down mounted on the vehicle to measure linear accelerations and angular velocities, providing high-frequency time and PVA estimates with high relative accuracy. Yet, as the absolute accuracy deteriorates along time when the INS is operating in stand-alone mode, the use of measurements provided by Global Position System (GPS) delivers high long-term stability to the coupled INS/GNSS solution (Rosales and Colomina, 2005).

The INS/GNSS integration scheme in SARVANT is based on a multi-step predictor-corrector method (Rosales and Colomina, 2005) to solve the INS stochastical dynamic system, and using an extended Kalman filter (EKF) approach for the update model. This method is highly convenient for non-uniform sampled IMU and GPS measurements; it is arbitrarily accurate and still compliant with real-time requirements for on-line processing.

Sensors and acquisition system. The acquisition system main components consist of:

o A NovAtel receiver OEM3, which specifications are summarized in Table 1.

o An Inertial Science's DMARS I, featuring Dynamically Tuned Gyroscopes (DTG) and pendulous linear accelerometers, it is of small size and low power consumption. Its nominal specifications are summarized in Table 2.

o A timing board which is synchronized with GPS time.

Table 3: NovAtel receiver specifications

\begin{tabular}{|l|l|}
\hline \multirow{3}{*}{ Position Accuracy } & DGPS: $0.45 \mathrm{~m}$ RMS \\
\cline { 2 - 2 } & OmniSTAR: VBS 0.7 m RMS \\
\cline { 2 - 2 } & CDGPS: $0.6 \mathrm{~m}$ RMS \\
\hline Velocity Accuracy & $0.03 \mathrm{~m} / \mathrm{s}$ RMS \\
\hline
\end{tabular}

Table 4: DMARS-I IMU specifications

\begin{tabular}{|l|l|}
\hline Size & $4.3 "$ diameter $\times 4.5^{\prime \prime ~ h}$ \\
\hline Weight & $5.5 \mathrm{lbs}$ \\
\hline Power & $22 \mathrm{Watts}$ \\
\hline Frequency & $200 \mathrm{~Hz}$ \\
\hline Linear: & \multicolumn{2}{|l|}{$200 \mu \mathrm{g}$} \\
\hline Bias & $10 \mu \mathrm{g}$ \\
\hline In-run bias stability & $10 \mu \mathrm{g} / \mathrm{VHz}$ \\
\hline Random noise & $200 \mathrm{ppm}$ \\
\hline Scale factor & \multicolumn{2}{|l}{} \\
\hline Angular: & $0.7 \mathrm{deg} / \mathrm{hr}$ \\
\hline Bias &
\end{tabular}

\begin{tabular}{|l|l|} 
In-run bias stability & $<0.3 \mathrm{deg} / \mathrm{hr}$ \\
\hline Random noise & $0.005 \mathrm{deg} / \sqrt{\mathrm{hr}}$ \\
\hline Scale factor & $200 \mathrm{ppm}$ \\
\hline
\end{tabular}

\section{FAST-AT ORIENTATION METHOD}

Recently (Blázquez and Colomina, 2012a) proposed an orientation method for large-format metric frame cameras that is far more accurate than Direct Sensor Orientation (DiSO) and almost as accurate as -yet less computationally costly thanIntegrated Sensor Orientation (ISO). The method assumes that INS/GNSS-derived position and attitude aerial control observations are available and that the cameras are precalibrated; i.e., that short before of after the flight the interior orientation elements and the radial/tangential distortions are determined using a calibration terrestrial test field. Fast AT applies no self-calibration to images but absorbs GNSS systematic errors and coordinate reference frame (GNSS to local) inconsistencies, and estimates the boresight system calibration parameters (Blázquez and Colomina, 2012a).

Since the SARVANT UAS is equipped with an INS/GNSS system featuring a high-end tactical- or navigation-grade IMU, the Fast AT method is fit for the purpose of computing orientation parameters for the SARVANT's optical remote sensing payload. If, in the future, due to camera calibration instabilities, Fast AT proves to be insufficient for the purpose, then the ISO method with INS/GNSS aerial control can always be used. In the ISO case, surface control observations as proposed in (Ebner et al., 1991) is an appealing complement, where the surface observations would be derived from the digital elevation model produced by the SARVANT's SAR payload and post-processing systems.

\subsection{Fast-AT: quasi-direct orientation}

Fast AT is a particular case of ISO characterized by the use of the following observations: tPA (time-Position-Attitude) aerial control observations for all images; ground control point observations for a limited number (in principle) of points and images; and image coordinate observations for the ground control points only.

A Fast AT block is simply an ISO block when all Tie Points (TPs) are Ground Control Points (GCPs) and where the number of GCPs is small. The concept is simple and can be performed with existing software with no or minimal modifications. Fast AT lies between DiSO and ISO, as it uses more observations than DiSO and fewer than ISO.

An advantage of Fast AT is that, with the exception of the image measurements for the GCPs, there are no further photogrammetric measurements to be made. This makes the method far less sensitive to poorly textured areas like deserts or rain forests and avoids the use of image matching software thus reducing costs and process time.

With rectangular blocks of large-format metric digital cameras, in average, Fast AT improves the accuracy (measured by the accuracy of ground point determination) of DiSO by $90 \%$ and consequently remains as close as $10 \%$ of ISO (we note that a factor of $0 \%$ with respect to DiSO means no improvement, and a factor of $100 \%$ or larger means equal or better performance than ISO) (Blázquez and Colomina, 2012a). For corridor 
blocks, the average improvement with respect to DiSO is at the $75 \%$ level, and thus the factor left to reach ISO is $25 \%$ (Blázquez and Colomina, 2012b).

\subsection{Application to the proposed system}

As mentioned before, the SARVANT aircraft navigation and orientation payload is typically equipped with a high-end tactical- or navigation-grade IMU because of the demanding requirements of X-band and, particularly, of the P-band onboard SAR. Together with a geodetic grade GPS receiver, the resulting INS/GNSS system guarantees a quality trajectory determination at the dm-accuracy level and at the 5 to $10 \mathrm{~cm}$ noise level (Fast AT is able to absorb the possible systematic errors as discussed above). What then remains is to calibrate the optical payload off-line, "short" before or after the flight where "short" is a function of the geometric stability of the cameras. For this purpose a terrestrial calibration field is established (signalized 2D or 3D calibration field) with coded (for automation purposes) and precise-pointing targets. Some of the targets are surveyed so local accurate coordinates can be assigned to them. The calibration procedure follows then, as usual, by systematically acquiring images at different distances to the calibration field and with different attitude angles. Finally, the photogrammetric measurement of the targets followed by a bundle adjustment with simultaneous estimation of the Conrady-Brown 5-parameters and the camera interior orientation parameters concludes the calibration procedure.

\section{CONCLUSIONS AND OUTLOOK}

This paper presents the SARVANT system, a UAV-based aerial remote sensing and mapping concept featuring a SAR able to sense in two different bands (X- and P-band) and a high-quality INS/GNSS navigation system. This system is able to explore small areas (up to 500 squared kilometres) to produce DTM of densely forested areas. In addition, the concept will be enhanced with an optical payload, based on a double-camera configuration to capture the visible and NIR spectrum for cartographic, environmental, forest and agricultural applications.

On the operability, the next steps deal with performing exhaustive testing in areas with corresponding authorization from the regulatory agency (nowadays, it is waiting for approval). The test sequence involves auto-pilot testing, payload remote control, data acquisition tests and data processing. Upon successfulness of this testing phase, the first SARVANT imagery will be available and data processing will enable the orientation of such imagery.

\section{REFERENCES}

Bendig, J., Bolten, A., Bareth, G., 2012. Introducing a LowCost Mini-UAV for Thermal- and Multi-spectral imaging, 2012. International Archives of the Photogrammetry, Remote Sensing and Spatial Information Sciences, Volume XXXIX-B1, XXII ISPRS Congress, 25 August 01 September 2012, Melbourne, Australia.

Blázquez, M., Colomina, I., 2012a. Fast AT: a simple procedure for quasi direct orientation. ISPRS Journal of Photogrammetry and Remote Sensing Vol. 71, No. 1, pp. 1-11.
Blázquez, M., Colomina, I., 2012b. Performance analysis of Fast AT for corridor aerial mapping. International Archives of Photogrammetry, Remote Sensing and Spatial Information Sciences, 2012-08-25-09-01, Melbourne, Australia.

Braga, R.T.V., Branco, K.R.L.J.C., Trindade Jr., O., Masiero, P.C., Neris, L.O., Becker, M, 2012. The ProLICES approach to develop product lines for safety-critical embedded systems and its application to the unmanned aerial vehicles domain. CLEI Electronic Journal 15(2), 1-13 (2012).

C-Astral Ltd., 2013. The C-ASTRAL BRAMOR gEO flies a mapping mission at $4150 \mathrm{~m}$ ASL.

$\mathrm{http}: / / \mathrm{c}$-astral.com/the-c-astral-bramor-geo-flies-a-mappingmission-at-4150m-asl (visited 20-May-2013).

Cho, G. et al., 2013. Pilotless aerial vehicle systems: size, scale and functions. Coordinates, Vol. 9, No. 1, pp. 8-16.

Ebner, H., Strunz, G., Colomina, I., 1991. Block triangulation with aerial and space imagery using digital terrain models as control information. ASP-ACSM Convention, 1991.3.25-29, Baltimore (Maryland).

Eisenbeiss, H., 2009. UAV photogrammetry. Diss. ETH No. 18515, doi:10.3929/ethz-a-005939264

Manyoky, M., Theiler, P., Steudler, D., Eisenbeiss, H., 2011. Unmanned Aerial Vehicle in cadastral applications. International Archives of the Photogrammetry, Remote Sensing and Spatial Information Sciences, Vol. XXXVIII-1/C22 UAV-g 2011, Conference on Unmanned Aerial Vehicle in Geomatics, Zurich, Switzerland.

MundoGEO, 2013. Anac concede primeiro certificado para VANT privado e civil no Brasil.

http://mundogeo.com/blog/2013/05/29/anac-concede-primeirocertificado-para-vant-privado-e-civil-no-brasil (visited 20-May2013)

Petrie, G., 2013. Commercial operation of lightweight UAVs for aerial imaging and mapping. GEOInformatics, Vol. 16, No. 1, pp. 28-39.

Remondino, F., Barazzetti, L., Nex, F., Scaioni, M., Sarazzi, D., 2011. UAV photogrammetry for mapping and $3 \mathrm{~d}$ modeling current status and future perspectives-. International Archives of the Photogrammetry, Remote Sensing and Spatial Information Sciences, Vol. XXXVIII-1/C22 UAV-g 2011, Conference on Unmanned Aerial Vehicle in Geomatics, Zurich, Switzerland.

Remy, M., de Macedo, K.A.C., Moreira, J., 2012. The first UAV-based P- and X-band interferometric SAR system. Intl. Geos. And remote Sensing Symp. (IGARSS), Munich, Germany, Jul. 2012.

Rosales, J.J., Colomina, I. 2005. A flexible approach for the numerical solution of the INS mechanization equations. Proceedings of the 6th Geomatic week, Barcelona, 2005.

Rufino, G., Moccia, A., 2005. "Integrated VIS-NIR Hyperspectral / Thermal-IR Electro-Optical Payload System for a Mini-UAV," AIAA 5th Aviation, Technology, Integration, and Operations Conference 26-29 September 2005, Arlington, Virginia. 
Strecha, C., Fletcher, A., Lechner, A., Erskine, P., Fua, P., 2012. Developing species specific vegetation maps using multispectral hyperspatial imagery from Unmanned Aerial Vehicles. International Archives of Photogrammetry, Remote Sensing and Spatial Information Sciences, 2012-08-25-09-01, Melbourne, Australia.

Vallet, J., Panissod, F., Strecha, C., Tracol, M., 2011. Photogrammetric performance of an ultra light weight swinglet "UAV". International Archives of the Photogrammetry, Remote Sensing and Spatial Information Sciences, Vol. XXXVIII1/C22 UAV-g 2011, Conference on Unmanned Aerial Vehicle in Geomatics, Zurich, Switzerland.

Wester-Ebbinghaus, W., 1980. Aerial Photography by radio controlled model helicopter. The Photogrammetric Record, Vol. 10, No. 55, pp. 85-92.

Zarco-Tejada, P.J., Berni, J.A.J., 2012. Vegetation monitoring using a micro-hyperspectral imaging sensor onboard an Unmanned Aerial Vehicle (UAV). Proceedings of the European Calibration and Orientation Workshop (EuroCOW), 7-10th February 2012, Barcelona, Spain . 